\title{
Hyperpigmentation Associated with Anti-Tumor Chemotherapy: A Series of 54 Cases, Demo-Topographic Aspects and Interest of the Dermatological Intervention
}

\author{
Tarik Hanafi", Hicham Titou, Hasna Kerrouch, Rachid Frikh, Naoufal Hjira and Mohammed Boui
}

Department of dermatology and venereology, Mohammed V Military Hospital, Faculty of Medicine and Pharmacy of Rabat, Mohammed V University, Morocco

*Corresponding author: Tarik Hanafi, Department of dermatology and venereology, Mohammed V Military Hospital, Faculty of Medicine and Pharmacy of Rabat, Mohammed V University, Hay Riad 10000 Rabat, Morocco

\begin{abstract}
Goals

1. Establish demographic and topographic mapping of hyperpigmentation's associated with antitumor chemotherapy.

2. Assess the psychological impact of hyperpigmentation in cancer patients and the interest of the dermatological intervention.
\end{abstract}

Methods: Three-year prospective study, from April 2016 to May 2019, including patients followed in oncology for malignant neoplasia, who developed hyperpigmentation following the start of antitumor chemotherapy for digestive, mammary and hematologic neoplasms, including the following molecules: 5 Fluorouracil, Tegafur, Cyclophosphamides, and Doxorubicin, in an isolated or combined mode.

1. Collection of demographic and topographical data.

2. Assessment of MELASQOL before and 3 months after dermatological intervention.

Results

1. The study enrolled 54 patients, with an average age of 48 years and extremes ranging from 22 years to 67 years, with a sex ratio $\mathrm{M} / \mathrm{W}$ of 1.2 . The most affected phototypes were III and IV which accounted for $63 \%$ of cases. The cutaneous involvement was largely dominant $83.3 \%$, the melanonychias of the fingers and/or toes were observed in 10 patients (18.5\% of the cases), the pigmentation of the oral mucosa in 2 patients, and genital in one patient. The average time to cutaneous involvement was six weeks with variables ranging from one day to five months, lesions were most often localized $(64.8 \%)$, diffuse in $(22.2 \%)$, circumscribed at injection sites in 07 patients. It affects photo-exposed areas in $74 \%$ of cases.
2. Through the evaluation of MELASQOL, we have shown that these hyperpigmentation's associated with a high MelasQol score can be improved by dermatological intervention to ensure a better quality of life for cancer patients.

\section{Keywords}

Chemotherapy, Hyperpigmentation, MELASQoL

\section{Introduction}

Hyperpigmentation as a side effect of anti-tumor chemotherapy is a frequent and usually mild complication [1], several molecules have been implicated based on different pathogenic theories [2]. However, studies that have focused on this complication are rare. Indeed, the benignity of the incident contrasting with the gravity of the tumor prognosis traces a mixed zone between the consideration of the oncologist and that of the cancer patient who perceives a display hyperpigmentation against a discreet internal neoplasia, this study will draw a demographic, topographical and targeted mapping of this complication, and will assess the psychological impact of hyperpigmentation in cancer patients as well as the interest of the dermatological intervention. It is believed that the dermatologist could contribute to improve the experience of cancer patients thanks to an anticipative, informative and pragmatic approach.

\section{Materials and Methods}

We ran a prospective study from April 2016 to May 2019, the inclusion criteria were, a follow-up for ma-

Citation: Hanafi T, Titou H, Kerrouch H, Frikh R, Hjira N, et al. (2020) Hyperpigmentation Associated with Anti-Tumor Chemotherapy: A Series of 54 Cases, Demo-Topographic Aspects and Interest of the Dermatological Intervention. Int J Cancer Clin Res 7:130. doi.org/10.23937/2378-3419/1410130 Accepted: January 25, 2020; Published: January 27, 2020

Copyright: (C) 2020 Hanafi T, et al. This is an open-access article distributed under the terms of the Creative Commons Attribution License, which permits unrestricted use, distribution, and reproduction in any medium, provided the original author and source are credited. 
lignant neoplasms (breast, digestive or hematologic neoplasms), a chemotherapy regimen including, 5 Fluorouracil, Tegafur, cyclophosphamides or Doxorubicin, in an isolated or combined mode, and finally hyperpigmentation installed after the start of chemotherapy protocol. Patients who met these criteria were referred to dermatology consultation from the oncology department. We excluded from this study patients having received other molecules before the onset of hyperpigmentation, and patients with non-melanin dyschromia.

We collect demographic (Age, sex and Phototype) and topographic data (location and extension).

The skin phototype was determined according to the Fitzpatrick scale $[3,4]$ (I) White skin, always burns, never tans; II) White skin, always burns, minimal tan; III) White skin, burns minimally, tans moderately and gradually; IV) Light brown skin, burns minimally, tans well; V) Brown skin, rarely burns, tans deeply; VI) Dark brown/ black skin, never burns, tans deeply).

The psychological impact was assessed using the MelasQol [5] score, the Arabic version [6] of which was recently validated. The value of the dermatological intervention was evaluated by carrying out a second MELASQOL score three months after the first consultation.

The dermatological intervention consisted of: IEA approach (information, education and awareness), particularly with regard to the benign and resolving nature of lesions, Photoprotection measures, Minimalist cosmetic intervention and corrective medical make-up.

\section{Results}

A total of 82 patients were referred from the medical oncology department to the dermatology consultation. Twenty-eight of them were excluded from this study, because they had already received other chemotherapy molecules, or other treatments started recently, less than three months before the onset of hyperpigmentation, or in whom clinical examination led to suspect another differential diagnosis. Thus, only fifty-four patients were included in the study, distributed as follows, Breast cancer (8 cases), gastric adenocarcinoma (12 cases), colorectal cancer (17 cases), Hodgkin lymphoma (9 cases), Non-Hodgkin lymphoma (5 cases), Leukemia (1 case), mycosis fungoides ( 2 cases), with an average age of 48 years and extremes varying from 22 years to 67 years, a sex $M / W$ ratio of $1: 2$. The most affected Phototypes were III and IV which represented $63 \%$ of the cases. The expression was heterogeneous; however, the skin involvement was largely dominant $83.3 \%$, melanonychia of the fingers and/or toes were observed in 10 patients ( $18.5 \%$ of cases), pigmentation of the oral mucosa in 2 patients and genital in one patient. The average time for skin involvement was six weeks with variables ranging from one day to five months. Most often localized (64.8\%), diffuse in (22.2\%), lim-
Table 1: Demographic and topographic mapping.

\begin{tabular}{|l|l|}
\hline & Total $\mathbf{n}=\mathbf{5 4}$ \\
\hline Age in years. Average (range) & $48(22 ; 67)$ \\
\hline Sex n (\%) & \\
\hline Man & $29(53)$ \\
\hline Woman & $25(46)$ \\
\hline I & \\
\hline II & $06(11.1)$ \\
\hline III & $09(16.6)$ \\
\hline IV & $16(29.6)$ \\
\hline V & $18(33.3)$ \\
\hline VI & $03(5.5)$ \\
\hline Location $\mathbf{n}$ (\%) & $02(3.7)$ \\
\hline Cutaneous & \\
\hline Nail & $45(83.3)$ \\
\hline Mucous & $10(18.5)$ \\
\hline Topography $\mathbf{n}(\%)$ & $03(5.5)$ \\
\hline Localized & \\
\hline Diffuse & $35(64.8)$ \\
\hline Restricted to injection sites & $12(22.2)$ \\
\hline Photo-exposed areas & $07(12.9)$ \\
\hline
\end{tabular}

ited to injection sites in 07 patients. It affected the photo-exposed areas in $74 \%$ of the cases (Table 1 ).

The initial evaluation of the MelasQol score (QUALITY OF LIFE) objectified an average value of 28/70 (varying from 10 to 38), particularly for display disorders.

The interest of the therapeutic dermatological intervention was evaluated by the appreciation of the change of MELASQOL (Figure 1)

- Group I: A clear improvement of more than $75 \%$ in (MELASQOL) in 06 patients (15\%)

- Group II: An improvement between $25 \%$ and $75 \%$ of (MELASQOL) in 40 patients (74\%)

- Group III: An aggravation or improvement of less than $25 \%$ in (MELASQOL): in 08 patients (11\%).

\section{Discussion}

The clinical expression of hyperpigmentations secondary to anti-tumor chemotherapy remains very heterogeneous [7], This heterogeneity could be systematized by opposing the diffuse forms $[8,9](22.2 \%$ of the cases in our study) with focal forms (64.8\%), including palmoplantar localization [10] and/or on areas of trauma or friction [11] and/or folds [12], as well as the other forms known as circumscribed at the injection sites [13] (12.9\% of cases), which are frequently reported in the literature, although they are represented in small proportion in our cohort, we believe that these circumscribed forms were not displayed to cause social discomfort and/or were 


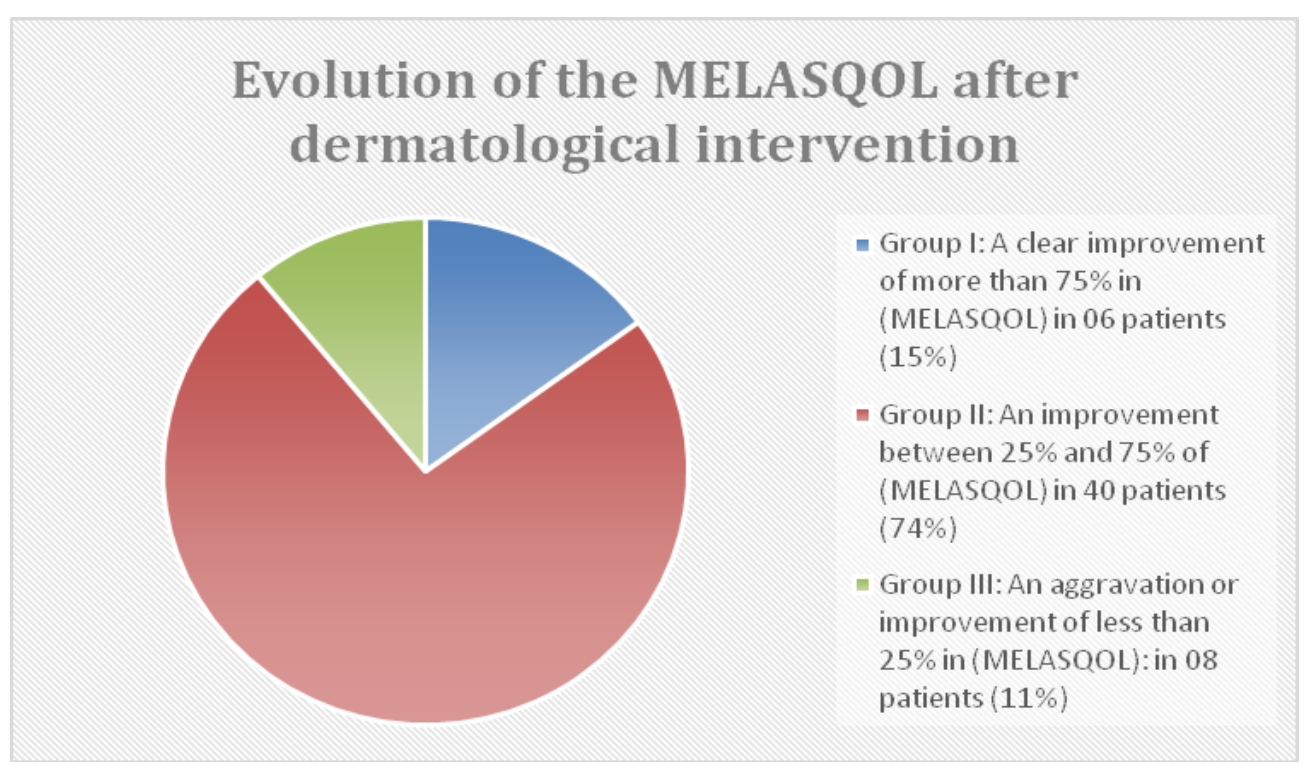

Figure 1: Evolution of the MELSQOL after the dermatological intervention.

not worrying to justify dermatological control in our patients, unlike the forms on photo-exposed areas (74\%) which were dominant. In addition, nail involvement remains a frequent incident in the form of pigmented bands [14] or diffuse pigmentation [8], unlike mucosal involvement which is rarer [15].

This clinical expression is variable depending on the molecule, the dose administered and the route of administration [8], these observations have suggested several pathogenic theories without being conclusive, thus certain molecules would act by direct stimulation of the melanogenesis [10] by endocrinological anomalies of the secretion of ACTH (Adreno CorticoTrophic Hormone) and $\alpha-\mathrm{MSH}$ ( $\alpha$-Melanocyte-stimulating hormone), Other authors suggest a direct toxic effect on epidermal melanocytes stimulating the increase melanin production [2], pigment incontinence [16] or even post-inflammatory hyperpigmentation [8]. The preferential location in certain microtrauma or occlusal areas could be explained by a local increase in blood flow or eccrine excretion prolonging the contact time between the molecule and the melanocytes $[2,8]$.

In 2018, Pollo, et al. [17] evaluated all the tools reported in the literature to measure the impact of melasma on quality of life, this study identified only one specific instrument for this aim, the MELASQOL [5].

MELASQOL [5] is currently the most commonly used tool to assess the impact of melasma on the daily lives of patients, particularly in relation to the three areas that seem to be the most affected, notably social life, leisure and emotional well-being. This score can help in the therapeutic decision but also will allow evaluating the effectiveness of possible treatments.

Several adaptations translated into different languages have been proposed and validated $[6,18,19]$, however these adaptations were based on the com- position of other questionnaires and not on the individual symbolic perceptions of patients [17], this defect in psychometric construction suggests a certain intercultural maladjustment.

In the absence of other tools, we have used MELASQOL in our cancer patients, to assess the impact of hyperpigmentation on their daily lives, but also to assess the impact of the dermatological intervention. This score has proven useful, particularly for display and photo-exposed forms, objectifying a real impact on quality of life, however the psychometric evaluation of our patients remains delicate under the shadow of an active malignant neoplasia, which is limiting the interest of MELASQOL.

\section{Conclusion}

We have provided a demo-topographic mapping of this complication, knowing that the data in the literature is limited to reports of isolated cases.

Through MELASQOL, we have proven that these pigmentary manifestations correlated with antitumor chemotherapy, and associated with a high MelasQol score, can be improved ensuring a better quality of life for cancer patients, in particular by simple measures of generous information, photoprotection, or in some cases, by a minimalist topical depigmenting treatment within the limits of safety and/or a corrective medical make-up.

\section{References}

1. Sibaud V, Fricain J-C, Baran R, Robert C (2013) Pigmentary disorders induced by anticancer agents. part I: Chemotherapy. Ann Dermatol Vénéréol 140: 183-196.

2. Wendy S Susser, Diane L Whitaker-Worth, Jane M GrantKels (1999) Mucocutaneous reactions to chemotherapy. Journal of the American Academy of Dermatology 40: 367398. 
3. Fitzpatrick TB (1988) The validity and practicality of sunreactive skin types I-VI. Arch Dermatol 124: 869-871.

4. Roberts WE (2009) Skin type classification systems old and new. Dermatol Clin 27: 529-533.

5. Balkrishnan R, McMichael AJ, Camacho FT, Saltzberg F, Housman TS, et al. (2003) Development and validation of a health-related quality of life instrument for women with melasma. Br J Dermatol 149: 572-577.

6. Doaa AE Abou-Taleb, Eman MK Youssef, Ahmed K Ibrahim, Alaa EA Moubasher (2014) Reliability and validity of the Arabic version of the Melasma Quality of Life questionnaire: (MELASQoL-A) study. 2: 121-127.

7. Didier Bessis, Camille Francès, Bernard Guillot, JeanJaques Guilhou (2009) Manifestations dermatologiques des maladies du système hématopoïétique et oncologie dermatologique : Dermatologie et médecine 3.

8. Bronner A, Hood A (1983) Cutaneous complications of chemotherapeutic agents. J Am Acad Dermatol 9: 645-663.

9. Muggia FM, Hainsworth JD, Jeffers S, Miller P, Groshen S, et al. (1997) Phase II study of liposomal doxorubicin in refractory ovarian cancer: Antitumor activity and toxicity modification by liposomal encapsulation. J Clin Oncol 15: 987-993.

10. C Vázquez-Bayo, AL Rodríguez-Bujaldón, R JiménezPuya, M Galán-Gutiérrez, JC Moreno-Giménez (2007) Capecitabine Induced Hyperpigmentation. Actas Dermosifiliogr 98: 491-493.

11. Horn T, Beveridge R, Egorin M, Abeloff M, Hood A (1989) Observations and proposed mechanism of $\mathrm{N}, \mathrm{N}^{\prime} \mathrm{N}$ "-triethylenethiophosphoramide (thiotepa)-induced hyperpigmentation. Arch Dermatol 125: 524-527.
12. Prussick R, Horn T, Wilson W, Turner M (1996) A characteristic eruption associated with ifosfamide, carboplatin, and etoposide chemotherapy after pretreatment with recombinant interleukin-1. J Am Acad Dermatol 35: 705-709.

13. Vukelja S, Bonner M, McCollough M, Cobb P, Gaule D, et al. (1991) Unusual serpentine hyperpigmentation associated with 5-fluorouracil: Case report and review of cutaneous manifestations associated with systemic 5-fluorouracil. J Am Acad Dermatol 25: 905-908.

14. Gropper C, Don P, Sadjadi M (1993) Nail and skin hyperpigmentation associated with hydroxyurea therapy for polycythemia vera. Int J Dermatol 32: 731-733.

15. Michael Lawrenz Co, Marcus Juan Esteban (2017) Lingual hyperpigmentation after 5-fluorouracil chemotherapy. BMJ Case Rep.

16. Schallier D, Decoster L, de Greve J (2011) Pemetrexed-induced hyperpigmentation of the skin. Anticancer Res 31: 1753-1755.

17. Pollo CF, Meneguin S, Miot HA (2018) Evaluation Instruments for Quality of Life Related to Melasma: An Integrative Review. CLINICS 73: e65.

18. Dominguez AR, Balkrishnan R, Elizey AR, Pandya AG (2006) Melasma in Latina patients: cross-cultural adaptation and validation of a quality-of-life questionnaire in Spanish language. J Am Acad Dermatol 55: 59-66.

19. Dogramaci AC, Havlucu DY, Inandi T, Balkrishnan R (2009) Validation of a melasma quality of life questionnaire for the Turkish language: the MelasQoL-TR study. J Dermatolog Treat 20: 95-99. 